\title{
Comportamiento de las Muertes Violentas en Bogotá, 1997-2003
}

\author{
Ricardo Sánchez ${ }^{1}$, Paola Tejada ${ }^{2}$ y Jorge Martínez ${ }^{3}$ \\ ${ }^{1}$ Médico. Especialista en Psiquiatría. M. Sc. Epidemiología clínica. Especialista en \\ Estadística. Facultad de Medicina, Universidad Nacional de Colombia. E-mail: \\ rsanchezpe@unal.edu.co. \\ ${ }^{2}$ Médica. Universidad Nacional de Colombia. \\ E-mail: paola_andreat@hotmail.com \\ ${ }^{3}$ Licenciado en Matemáticas. Ph. D. Estadística. Departamento de Estadística, Facultad de \\ Ciencias, Universidad Nacional de Colombia. E-mail: jmartinezc@unal.edu.co
}

Recibido 10 Junio 2005/Enviado para Modificación 17 Septiembre 2005/Aceptado 3 Octubre 2005

\section{RESUMEN}

Objetivo Describir los patrones que muestran las muertes violentas en Bogotá durante 1997 a 2003

Diseño Se aplicaron técnicas de análisis de series de tiempo a 84 observaciones correspondientes a casos de mortalidad por causas violentas durante 7 años en Bogotá. Se efectuó modelamiento con la metodología de BoxJenkins y se generó un pronóstico de la serie a 12 meses para prever su tendencia. Fuentes de información primaria y secundaria: Instituto de Medicina Legal de Bogotá y Departamento Administrativo Nacional de Estadística de Colombia

Resultados Para cuatro tipos de muerte violenta analizados (homicidio, accidente de tránsito, suicidio y accidental diferente de accidente de tránsito), se observó una tendencia en la reducción del número de muertes durante el período analizado, más marcada en los casos de homicidio y accidente de tránsito. En todos los casos, excepto en los de suicidio, se evidenció un patrón estacional, con incremento de frecuencia de muertes en diciembre. Los pronósticos señalan un sostenimiento de las tendencias decrecientes de las series, más marcado en el caso de homicidios y accidentes de tránsito, que en el de suicidios y otras muertes accidentales.

Conclusiones En Bogotá, la mortalidad por causas violentas ha presentado una tendencia a la disminución. En el caso de suicidios y muertes accidentales diferentes de accidente de tránsito la frecuencia de muertes tiende a estabilizarse. Se plantea la realización de estudios que evalúen el papel de diferentes factores sobre esta dinámica de las muertes violentas. 
Palabras Claves: Series de tiempo, violencia, homicidio, suicidio (fuente: DeCS, BIREME).

\section{ABSTRACT \\ Patterns of violent death in Bogotá, 1997-2003}

Objective To describe patterns of violent deaths in Bogotá between 1997 and 2003.

Design Descriptive methods for analyzing time series were applied to a group of 84 observations corresponding to mortality due to violent causes during seven years in Bogotá. Box-Jenkins methodology was used to generate forecasts for 12 months. Primary and secondary sources of information: Instituto de Medicina Legal Bogotá, Departamento Administrativo Nacional de Estadística, Colombia

Results For the 4 types of violent deaths analyzed (homicide, transit accident, suicide and others accidents), a decreasing tendency in the number of deaths during the analyzed period was observed, more evident in homicide and transit accidents. In all cases, except in the group of suicides, evidence of seasonality, with an increase in frequency of deaths in December, has been observed. The forecasting suggests persistence of decreasing trends, more apparent in the series of homicide and transit accidents.

Conclusions In Bogotá, the mortality caused by violence has presented a decreasing trend. Stabilization of trend is observed in cases of suicides and accidental deaths. Further study is required to characterize and evaluate the contribution of different factors in this dynamic of violent death.

Key Words: Time series, violence, homicide, suicide (source: MeSH, NLM).

$\mathrm{E}$ n términos generales la violencia agrupa todo tipo de acciones que atentan contra la integridad personal y material de una sociedad (1). Este fenómeno abarca múltiples situaciones como homicidios, violencia intrafamiliar, asaltos, hurtos, acciones terroristas, etc. Por esto a la hora de estudiarlo se deben delimitar los tipos de violencia que se incluyan en el análisis.

Para el presente análisis se adoptará una definición operacional: La violencia es la manifestación de conflictos y condiciones sociales en los cuales se hace evidente la negligencia social para proteger la vida (2).

Es importante reconocer la violencia urbana como una forma de violencia diferenciada y, posiblemente, con determinantes específicos que la moldean y determinan (2). Por ejemplo, en el caso de los homicidios, las tasas urbanas han crecido a un ritmo superior al cincuenta por ciento de las tasas rurales 
(3). Por esto no es posible hablar en el país de una violencia, sino que nuestra sociedad experimenta múltiples formas de conflictos (3). En general, en Colombia hay deficiencia en el análisis riguroso de los fenómenos de violencia urbana (4), entre otras razones, porque las distintas instancias que recogen estos datos manejan la información pertinente sin confrontarla con las demás, utilizando muchas veces criterios y definiciones diferentes para registrar un hecho violento (1). Además, muchos investigadores tratan de analizar la violencia en la ciudad a partir de la misma óptica con la que se estudia la tradicional violencia política rural, o, en el caso de Bogotá, intentan equiparar las causas del fenómeno con las que lo potencian en ciudades como Cali o Medellín (5).

En Bogotá la violencia es un problema de primer orden: la principal causa de mortalidad en la población bogotana es producto de la violencia intencional (2). Además, se generan no sólo pérdidas de capital humano y físico sino costos económicos en diferentes sectores $(6,7)$. A diferencia de otras capitales latinoamericanas, Bogotá no es la más violenta y dentro del propio país tiene tasas inferiores a las de Cali y Medellín $(8,9)$. Otra particularidad es que la violencia no afecta por igual a toda la ciudad; por ejemplo, en el caso de los homicidios, estos están concentrados en pocas zonas, debido a que en ciudades grandes y heterogéneas como Bogotá los conflictos pueden ser dispersos y localizados en zonas particulares $(3,10)$.

El único indicador demográfico que, aunque de forma leve, ha mostrado cierta asociación con la tasa de homicidios, y de violencia en general, es el índice de masculinidad en el total de la población de una localidad $(4,10)$.

La prevención de la violencia tiene una doble dimensión: la objetiva, referida a los hechos concretos de violencia, y la subjetiva, que tiene que ver con la percepción y la representación que el ciudadano se hace de ella (11). Las actividades de prevención de la violencia involucran diversos sectores de la sociedad, campos científicos y organizaciones (12). En los últimos años se han hecho una serie de intervenciones para reducir la mortalidad por violencia en Bogotá. Estas acciones pueden dividirse en coercitivas y en programas de prevención de factores asociados a la violencia. Dentro de las primeras se encuentra el fortalecimiento de la Policía Metropolitana (13). En cuanto a las acciones preventivas, se han promulgado una serie de decretos y medidas siendo los de mayor impacto (14):

- Decreto 1193 de 1997 por el cual se prohibe el expendio de bebidas alcohólicas en droguerías y estaciones de servicio. 
- Decreto 909 de 2001 por el cual se adoptan medidas para la protección de menores de edad en el Distrito Capital de Bogotá (reglamenta que entre 11pm y 5 am los menores no pueden estar sin compañía de un adulto responsable en la vía pública o en establecimientos públicos).

- Decreto 207 de 1998 en el cual se establece el horario de funcionamiento de establecimientos para el expendio y consumo de bebidas alcohólicas en el territorio del Distrito Capital y se adoptan otras medidas.

- Decreto 751 de 2001 por el cual se adoptan medidas de control sobre la fabricación, almacenamiento, transporte, venta y manipulación de fuegos artificiales o artículos pirotécnicos en el Distrito Capital de Bogotá.

- Decreto 126 de 2002 por el cual se toman medidas para garantizar la seguridad de los habitantes del Distrito Capital y se dictan otras disposiciones.

- Julio 2003, campaña pedagógica "¿Bogotá, nos estamos acostumbrando, qué nos pasa?" impulsada por la Alcaldía Mayor y el Fondo de Prevención Vial.

Dado que la violencia es un fenómeno dinámico, este estudio pretende describir el comportamiento de algunas de sus manifestaciones y efectuar un pronóstico de la mortalidad por causas violentas en Bogotá a doce meses, utilizando una metodología de análisis de series de tiempo. Para tal efecto se consideran cuatro tipos de muerte violenta: homicidio, accidente de tránsito, suicidio y otras muertes accidentales diferentes de accidente de tránsito.

\section{MÉTODOS Y MATERIALES}

Este trabajo corresponde a un estudio observacional, ecológico, en el cual se utilizó la metodología propia del análisis estadístico de series de tiempo.

Se recolectaron datos de mortalidad mensual en Bogotá durante un período de siete años (se incorporaron datos desde 1997 a 2003). Para cada uno de estos años el total poblacional estimado de la ciudad fue (15): 
Los datos de mortalidad fueron suministrados por el Instituto de Medicina. Legal de Bogotá y se derivan de la información consignada en los protocolos de necropsias practicadas en esa institución. Esta fuente de datos se considera de alta calidad ya que la legislación colombiana obliga a practicar necropsia en casos de muerte violenta. El presente estudio consideró los casos en los que se confirmó una muerte violenta. Para el análisis de los datos se tuvo en cuenta la premisa de que, al referirse a problemas de salud pública, especialmente en lo relacionado con el número de vidas de seres humanos, puede resultar más apropiado manejar valores absolutos que relativos (16). Por tal motivo no se manejaron tasas. El análisis se efectuó con el programa Statgraphics ${ }^{\circledR}$, configurando series de 84 observaciones mensuales. El análisis de los datos implicó efectuar una distribución de frecuencias de las maneras de muerte según año; seguidamente se efectuó análisis de las series de tiempo que expresaban la frecuencia mensual de homicidios, suicidios, accidentes de tránsito y muertes accidentales diferentes de accidente de tránsito. El análisis de las series de tiempo tuvo un componente descriptivo y otro analítico. En el componente descriptivo se evaluaron las características generales de la serie para determinar la existencia de fuentes de variabilidad como tendencia, efectos estacionales, cambios cíclicos y variación aleatoria (17). Para ello se utilizó el método tradicional de descomposición multiplicativo de series partiendo de un suavizamiento de promedios móviles basado en el filtro de Spencer. A diferencia de los gráficos de la serie cruda (Figura 1) los de las series suavizadas (Figura 2) muestran con mayor claridad su tendencia. En los casos en que se descubrió una fluctuación estacional se calcularon índices estacionales para determinar la variación del número de muertes ocurrido a lo largo del año. Estos índices expresan en términos de porcentaje la relación existente entre un determinado mes y el promedio del año correspondiente. A partir de estos datos se generaron los gráficos de índices estacionales (Figura 3). Para el componente analítico se construyeron modelos ARIMA siguiendo la metodología de Box y Jenkins. Inicialmente se analizaron los autocorrelogramas de las series para determinar la estructura de autocorrelación de los datos y poder identificar un modelo para cada serie. Una vez estimados los parámetros de los modelos se realizaron las diferentes pruebas de bondad de ajuste recomendadas en estos casos. A partir del mejor modelo seleccionado se realizaron pronósticos para los 12 meses correspondientes al año 2004.

Los modelos considerados para el análisis de las series son de la forma:

$$
X_{t}=\theta_{0}+\phi_{1} X_{t-1}+\phi_{2} X_{t-2}+a_{t}-\theta_{1} a_{t-1}-\theta_{12} a_{t-12}
$$


donde $X_{t}$ es el número de muertes observadas en el mes $t$, o la variación mensual en los casos en que se encontró una clara tendencia de las series, $a_{t}$ es una variable que indica la componente aleatoria del modelo en el mes $t$, $\theta_{0}$ una constante relacionada con el valor promedio de la serie, $\phi_{1}$ y $\phi_{2}$ parámetros que incorporan en el modelo las muertes observadas en los meses anteriores y $\theta_{1}$ y $\theta_{12}$ parámetros con los cuales se considera en el modelo la estructura de autocorrelación no estacional y estacional.

\section{RESULTADOS}

Durante los siete años analizados las muertes por causa externa se distribuyeron así: accidente de tránsito 6873 (18,6\%), homicidio 16698 (45,3\%), suicidio $2343(6,4 \%)$, accidental diferente a accidente de tránsito 3204 (8,7\%), violenta por determinar 1017 (2,8\%), natural 3433 (9,3\%), indeterminada $237(0,6 \%)$, restos óseos encontrados sin determinar causa de muerte $54(0,2 \%)$ y en estudio $3015(8,2 \%)$. Esto indica que en el $88 \%$ de los casos la causa de muerte estuvo totalmente definida: $79 \%$ violentas y $9 \%$ naturales. En este estudio solamente se tomaron los casos de muerte por causa externa documentada (79\%). La distribución del tipo de muerte por causa externa según año se presenta en la Tabla 1. Para todos los años, el homicidio fue la manera de muerte por causa externa más frecuente.

Tabla 1. Frecuencia y porcentajes (\%) de muertes en Bogotá, 1997 a 2003.

\begin{tabular}{cccccccc}
\hline Manera & \multicolumn{7}{c}{ Años analizados } \\
\cline { 2 - 8 } de muerte & 1997 & 1998 & 1999 & 2000 & 2001 & 2002 & 2003 \\
\hline Accidente & 930 & 1173 & 1119 & 1032 & 940 & 883 & 796 \\
de tránsito & $(20,2)$ & $(18,5)$ & $(23,9)$ & $(18,3)$ & $(17,5)$ & $(16,7)$ & $(16,1)$ \\
& 2824 & 2697 & 2684 & 2426 & 2252 & 2057 & 1758 \\
Homicidio & $(61,4)$ & $(42,5)$ & $(57,4)$ & $(43,0)$ & $(41,9)$ & $(38,9)$ & $(35,6)$ \\
& 320 & 391 & 358 & 365 & 318 & 286 & 305 \\
Suicidio & $(7,0)$ & $(6,2)$ & $(7,7)$ & $(6,5)$ & $(5,9)$ & $(5,4)$ & $(6,2)$ \\
& 450 & 576 & 518 & 468 & 383 & 417 & 392 \\
Accidental & $(9,8)$ & $(9,1)$ & $(11,1)$ & $(8,3)$ & $(7,1)$ & $(7,9)$ & $(7,9)$ \\
no tránsito & $(9,8)$ & 6353 & 4679 & 5642 & 5371 & 5290 & 4940 \\
Total todas & 4599 & 6350 & $(100)$ & $(100)$ & $(100)$ & $(100)$ & $(100)$ \\
las causas & $(100)$ & $(100)$ & $(100)$ &
\end{tabular}

Los porcentajes se calculan sobre el total de todas las causas

Series de tiempo de muertes por homicidio 
Como lo muestra el gráfico de la serie (Figura 1A), el número de homicidios tiene una clara tendencia decreciente, con un rango de variación entre 113 y 289 casos y picos al final de cada año. La tendencia es aún más clara al observar la serie suavizada usando un filtro de amplitud 21 (Figura 2A). Las características de las estructuras de autocorrelación confirmaron la presencia de tendencia y de componente estacional.

El gráfico de los índices estacionales mostró incremento del número de homicidios en meses intermedios y al final del año, así como una disminución en los primeros meses del año (Figura 3A).

Figura 1. Series de tiempo de muertes por causa externa

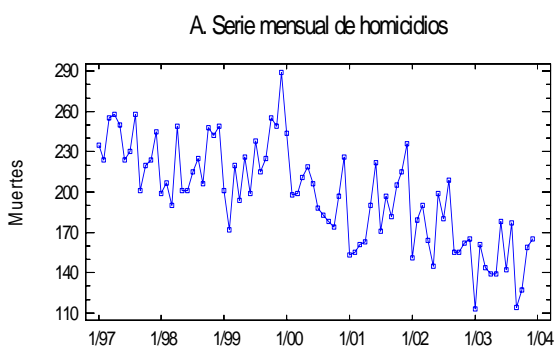

B. Serie mensual de accidentes de tránsito
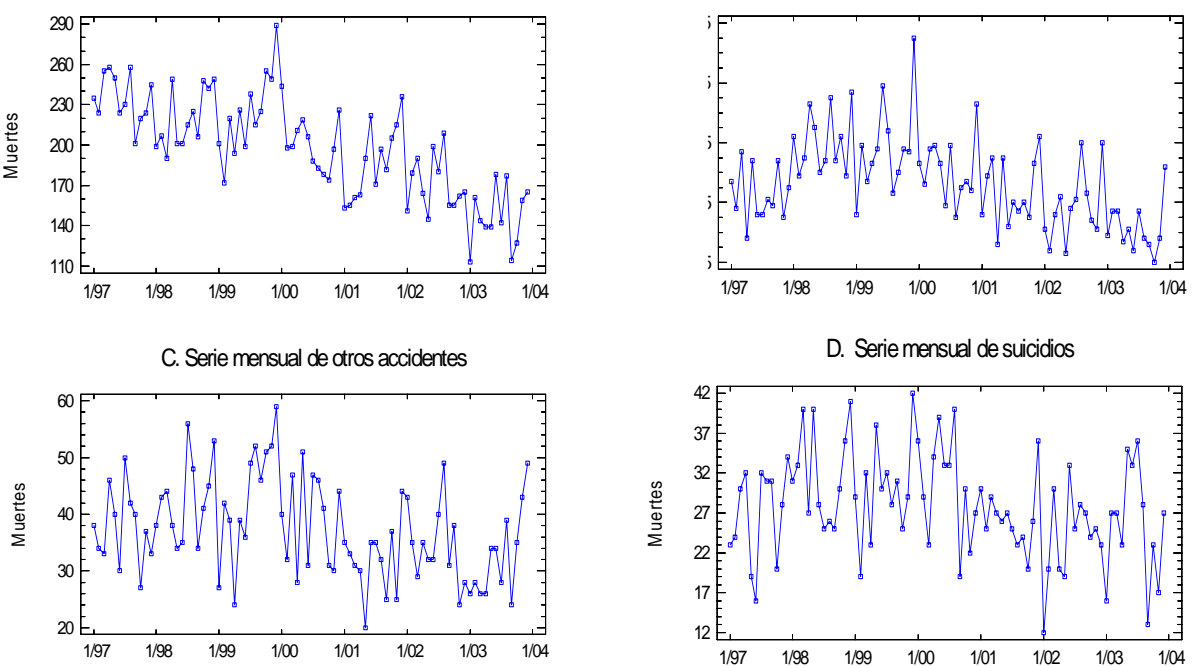

D. Serie mensual de suicidios

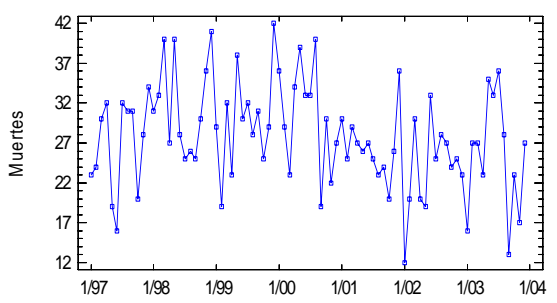

Esta serie, al igual que la de muertes por accidentes de tránsito y otras formas de accidente, presenta valores atípicos por su elevado nivel en diciembre de 1999.

Con base en la estructura de autocorrelación de la serie se planteó un modelo estacional del tipo ARIMA $(0,1,1) \times(0,1,1)_{12}$ definido como 


$$
\begin{gathered}
D_{t}=a_{t}-0,6423 a_{t-1}-0,8134 a_{t-12} \\
(0,0918)(0,0522)
\end{gathered}
$$

$D_{t}$ es una doble diferencia definida como $D_{t}=Y_{t}-Y_{t-12}$, siendo $Y_{t}=X_{t}-X_{t-1}$. Los números entre paréntesis corresponden a los errores estándar de las estimaciones de los parámetros del modelo.

Figura 2. Series de tiempo suavizadas
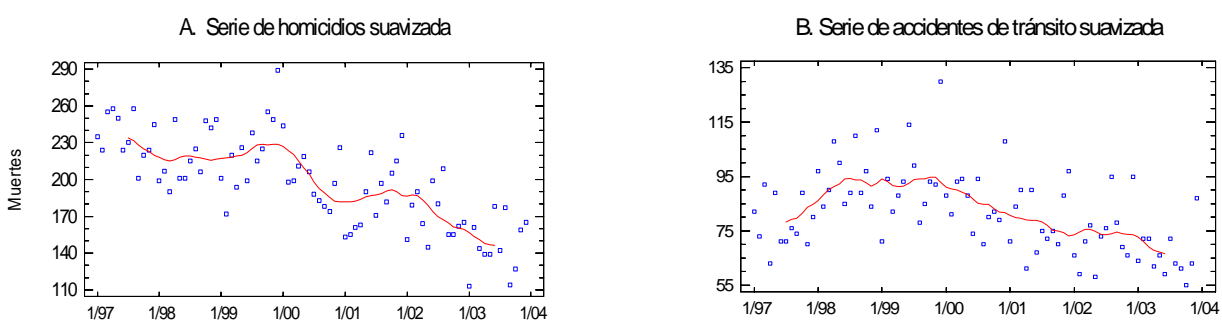

C. Serie de otros accidentes suavizada
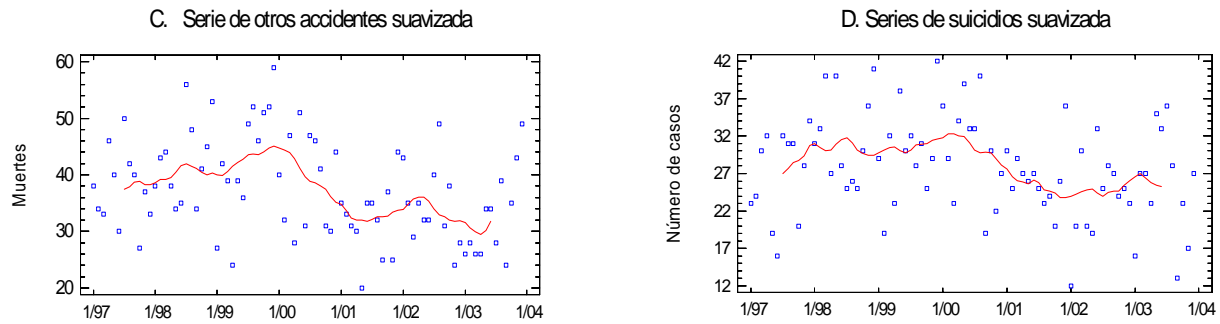

Figura 3. Características de los índices estaciónales
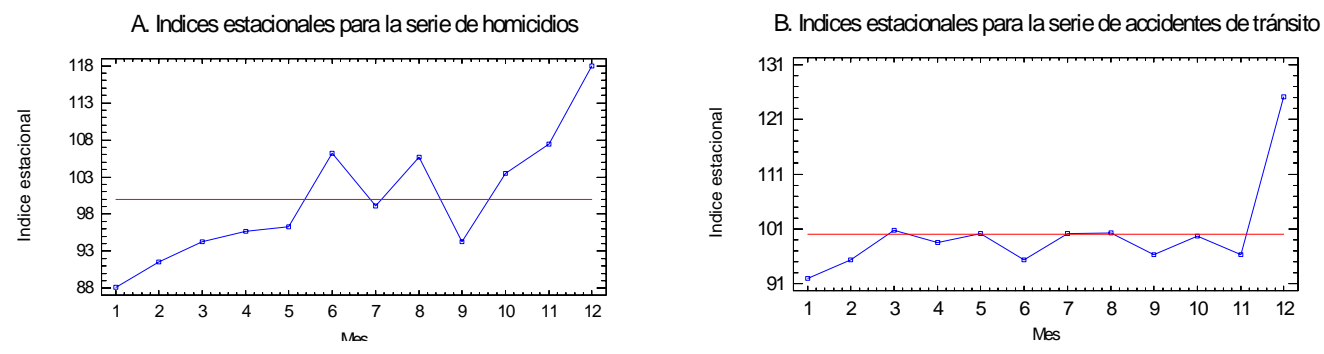

C. Indices estacionales para la serie de otros accidentes

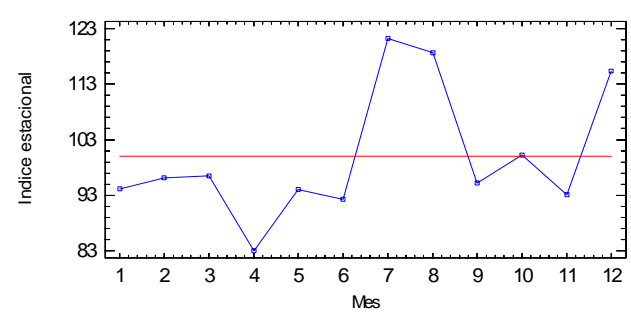


Para el diagnóstico del modelo se aplicaron pruebas estadísticas no paramétricas de aleatoriedad de los residuos del modelo, así como la prueba de Box-Pierce para determinar la no correlación de los mismos. Estas pruebas mostraron que el modelo propuesto cumple los supuestos requeridos para su validez. El autocorrelograma de residuos no mostró autocorrrelaciones significativas. La componente no explicada por el modelo tiene una desviación estándar de 22,8. Con base en el modelo propuesto se generaron pronósticos para los 12 meses del año 2004 los cuales muestran un mantenimiento de la tendencia decreciente (Figura 4A). Los pronósticos se presentan en la Tabla 2.

Figura 4. Series mensuales con pronósticos para 2004
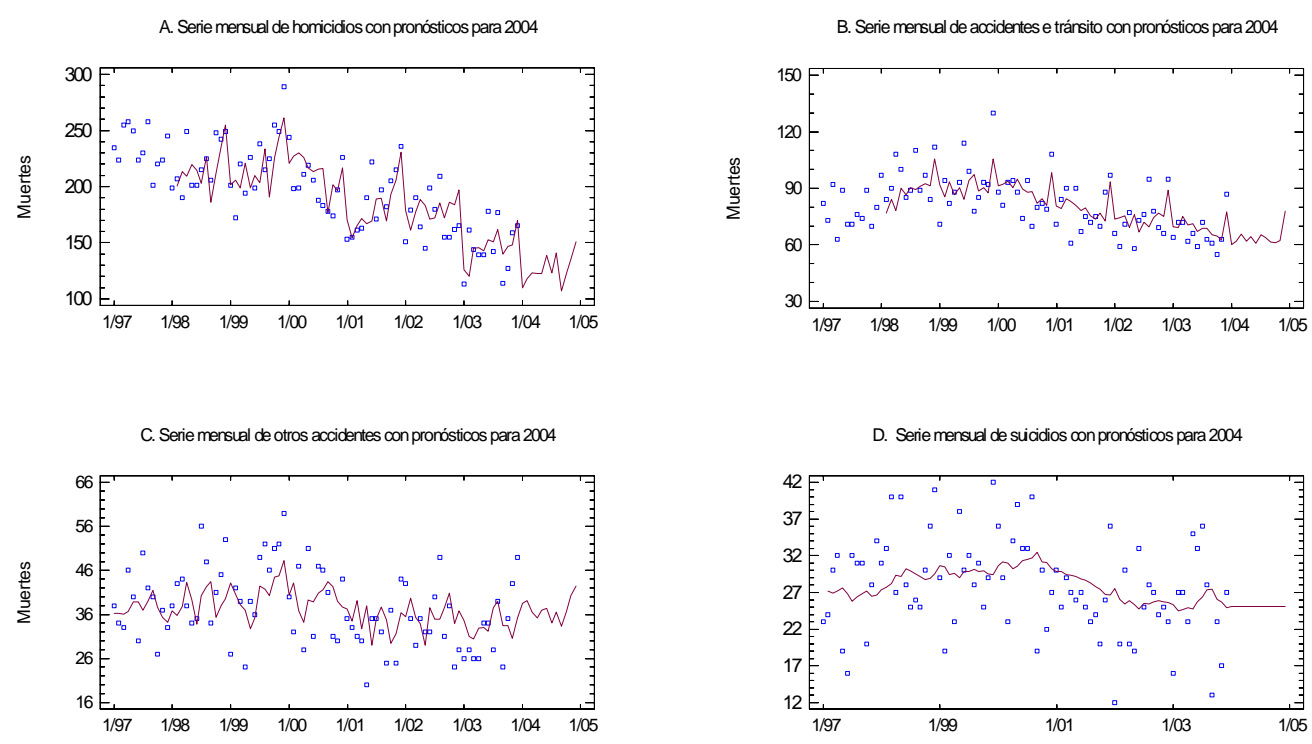

Series de tiempo de muertes por accidente de tránsito

Esta serie tiene un rango de variación entre 55 y 130 muertes y como lo muestra su gráfico (Figura 1B), a mediados del año 1999 se presenta un cambio notorio en su tendencia. La serie suavizada usando un filtro de amplitud 21 confirma la tendencia decreciente a partir de 1999 (Figura 2B) lo cual indica que debe estudiarse este fenómeno para determinar si la característica de la tendencia puede estar asociada a las medidas adoptadas en 1998. Las características de la estructura de autocorrelación indicaron la presencia de un componente estacional con comportamiento relativamente estable, ex- 
cepto en diciembre donde hay incremento del $25 \%$ sobre el promedio anual (Figura 3B).

Tabla 2. Pronósticos del número de muertes para el año 2004

\begin{tabular}{lcccc}
\hline \multicolumn{1}{c}{ Mes } & Homicidios & $\begin{array}{c}\text { Accidentes de } \\
\text { tránsito }\end{array}$ & $\begin{array}{c}\text { Otros acci- } \\
\text { dentes }\end{array}$ & Suicidios \\
\hline Enero & 110 & 60 & 39 & 28 \\
Febrero & 118 & 62 & 39 & 28 \\
Marzo & 123 & 66 & 37 & 28 \\
Abril & 123 & 62 & 35 & 28 \\
Mayo & 122 & 64 & 37 & 28 \\
Junio & 139 & 61 & 37 & 28 \\
Julio & 123 & 65 & 34 & 28 \\
Agosto & 141 & 64 & 37 & 28 \\
Septiembre & 107 & 62 & 33 & 28 \\
Octubre & 122 & 61 & 37 & 28 \\
Noviembre & 135 & 62 & 40 & 28 \\
\hline Diciembre & 151 & 78 & 42 & \\
\hline
\end{tabular}

A esta serie se le ajustó un modelo estacional del tipo $\operatorname{ARIMA}(0,1,1) \mathrm{x}(0,0,1)_{12}$ definido como

$$
\begin{gathered}
Y_{t}=a_{t}-0,8376 a_{t-1}+0,2052 a_{t-12} \\
(0,0576) \quad(0,1008)
\end{gathered}
$$

Aunque el modelo anterior presentó un ajuste razonable, se lograron mejores resultados con un suavizamiento estacional de Holt-Winters con parámetros $\alpha=0,201, \beta=0,000, \gamma=0,144$.

Para el diagnóstico del modelo se aplicaron pruebas de aleatoriedad no paramétricas y de Box-Pierce no encontrando violaciones significativas a los supuestos, lo cual sugiere que el modelo es adecuado. De otra parte el autocorrelograma de los errores no muestra valores significativos. La variable que expresa la variación aleatoria no explicada por el modelo tiene una desviación estándar de 12,6. Utilizando el anterior modelo se generó el pronóstico para los 12 meses del año 2004 que muestra la continuación de la tendencia decreciente y el pico al final del año (Figura 4B, Tabla 2).

Series de tiempo de muertes por accidentes diferentes de tránsito 
El gráfico de la serie (Figura 1C) no muestra una clara tendencia, aunque el suavizado muestra un patrón cíclico decreciente y un incremento en los últimos meses (Figura 2C). El rango de variación de la serie es entre 20 y 59 casos mensuales. Las características de las estructuras de autocorrelación sugirieron componente estacional. El gráfico de índices estacionales mostró un comportamiento con picos en los meses intermedios y a final del año (Figura 3C).

Para esta serie se consideró el modelo ARIMA $(2,0,0) \times(0,0,1)_{12}$ definido por

$$
X_{t}=20.1+0,2415 X_{t-1}+0,2182+a_{t}+0,3292 a_{t-12}
$$

Para el diagnóstico del modelo se utilizaron pruebas de aleatoriedad no paramétrica y de Box-Pierce, ninguna de las cuales mostró evidencia significativa de falta de aleatoriedad o autocorrelaciones de los residuos. Con base en el modelo planteado se generó un pronóstico para 12 meses que muestra una tendencia a la estabilización del número de las muertes accidentales (Figura 4C).

Series de tiempo de muertes por suicidio

La serie presentó un rango de variación de 12 a 42 muertes. El gráfico de la serie no evidencia claramente la presencia de tendencias ni de patrones cíclicos (Figura 1D), aunque la serie suavizada sí muestra leve tendencia cíclica decreciente desde el año 2000 (Figura 2D). Como la variabilidad de la serie no permite establecer de manera significativa la presencia de una tendencia, se ajustó el modelo ARIMA(1,0,0)

$$
\begin{gathered}
X_{t}=22.5+0,1897 X_{t-1}+a_{t} \\
(0,1008)
\end{gathered}
$$

Las pruebas de diagnóstico no muestran una significativa violación de los supuestos del modelo. Se generó un pronóstico para 12 meses que sugiere estabilidad en el comportamiento de los suicidios en Bogotá durante el año 2004 alrededor del promedio de 27,8 suicidios mensuales, con una desviación estándar de 6,4 (Figura 4d).

\section{DISCUSIÓN}


La mortalidad por causas externas da cuenta de una proporción importante de la mortalidad general en Bogotá. Esta elevada frecuencia relativa puede estar reflejando no solo un incremento real de la mortalidad por tal motivo, sino una reducción de las muertes por otras causas como las infecciosas o las relacionadas con otras patologías controlables o prevenibles. El estudio de los valores absolutos tendría la ventaja de mostrar la tendencia neta, sin la influencia de la dinámica de otras causas de muerte.

Se consideró que los registros de Medicina Legal son una fuente confiable para realizar el presente estudio. Un análisis preliminar de información disponible nos indicó que esta fuente es más confiable que la información disponible en el DANE. Por ejemplo, el número de casos de muerte por causa externa reportados por registros DANE en los años 1998, 1999 y 2000 fue de 4 824, 4753 y 4600 respectivamente, mientras que el número de registros por las mismas causas en Medicina Legal fue de 5 180, 4679 y 5642 para cada uno de esos años.

La mortalidad por causa externa es una situación de salud pública que puede ser intervenida para modificarla; se ha podido evidenciar que, por ejemplo, la mortalidad por accidentes de tránsito se ha reducido aplicando medidas como restricción del uso de bebidas alcohólicas, reducción del límite de velocidad o uso obligatorio del cinturón de seguridad (18). En Bogotá el impacto de medidas preventivas similares no se ha estudiado sistemáticamente. La aparente relación entre las fechas de aplicación de políticas específicas para la reducción de muertes violentas y el descenso evidenciado en las series debe evaluarse aplicando los métodos estadísticos apropiados. Hasta el momento no se han encontrado estudios con rigor metodológico que evalúen si la disminución en las frecuencias absolutas de muertes se relaciona con alguna medida de tipo punitivo o preventivo en el ámbito local. Por esta razón, se recomienda la realización de otros estudios que permitan dar respuesta a esta pregunta así como la incorporación de otras variables que podrían estar relacionadas, por ejemplo, de tipo económico. Estos estudios aportarían insumos que permitirían efectuar intervenciones para detectar y modificar factores de riesgo. Se sugiere la investigación de estas variables en subpoblaciones homogéneas ya sea por género, grupos de edad o pertenencia a una localidad de la ciudad. Debe reconocerse que en este estudio, al haber utilizado valores absolutos en lugar de tasas, se ha podido magnificar la tendencia a la reducción de las muertes.

De acuerdo con los hallazgos del estudio, a partir del año 2000 todos los tipos de muertes violentas presentan una tendencia a la disminución, más marcada en unos grupos que en otros. Como puede verse en las gráficas de 
series, los datos señalan una tendencia sostenida que, de acuerdo a las predicciones planteadas a partir de los modelos matemáticos, podrían estarse manteniendo hasta la actualidad.

Es llamativo el patrón estacional de diferentes tipos de muerte violenta, siendo consistente un aumento en el mes de diciembre de las muertes por accidentes de tránsito, de los homicidios y de las muertes accidentales, las cuales registran también un aumento en los meses de junio a agosto. A diferencia de otros datos reportados en estudios sobre conductas autolíticas en Bogotá (19), los suicidios no mostraron aumento significativo en una época del año en particular.

Agradecimientos. Al Instituto Nacional de Medicina Legal de Bogotá por los datos con los que se efectuó este análisis. A la División Nacional de Investigación de la Universidad Nacional de Colombia, por su apoyo financiero.

\section{REFERENCIAS}

1. Segovia G. La violencia en Santafé de Bogotá. Bogotá: Ecoe; 1994. p. 7-40.

2. Corporación Misión Siglo XXI. El futuro de la capital. Estudio prospectivo de seguridad. Bogotá: Cámara de Comercio de Bogotá; 1996. p. 17-141

3. Seminario Internacional: Violencia, Secuestro y Terrorismo (memorias). Bogotá; 1997. p 24-71.

4. Grupo de estudios Paz Pública del CEDE. Caracterización de la violencia homicida en Bogotá. Bogotá: Universidad de los Andes; 2000.

5. Seminario internacional sobre violencia urbana e inseguridad ciudadana (memorias) Santa Marta, 1994. p 134-140 .

6. Buitrago L. Bogotá, violencia difusa: un problema de salud pública. Bogotá: Universidad Nacional; 2000.

7. Gutiérrez, J. Violencia y criminalidad en Santa Fe de Bogotá: posibles determinantes y relaciones de doble causalidad. Bogotá: CEDE Universidad de los Andes; 2000.

8. Perczek R. Pobreza y violencia en Santafé de Bogotá. Bogotá: Universidad de los Andes; 1996

9. Acero H. Reducción de la violencia y la delincuencia en Bogotá, Colombia, 19942002. Biomédica 2002; 22:362-72.

10. Llorente M, Escobedo R, Escandía C, Rubio M. Violencia homicida en Bogotá: más que intolerancia. Bogotá: CEDE Universidad de los Andes; 2001.

11. Concha A. Impacto social y económico de la violencia en las Américas. Biomédica 2002; 22: 347-61.

12. Krug E, Mercy J, Dahlberg L, Zwi A. El informe mundial sobre la violencia y la salud. Biomédica 2002; 22: 327-36.

13. Sistema Unificado de Información de Violencia y Delincuencia en Bogotá. Acero H. Ciudad y políticas públicas de seguridad y convivencia [Internet]. 
Disponible en http://www.suivd.gov.co/ciudad/MexicoMarzohacero.doc Consultado Diciembre de 2004

14. Sistema Unificado de Información de Violencia y Delincuencia en Bogotá. Normas y Decretos [Internet]. Disponible en http://www.suivd.gov.co/ normas\%20y\%20decretos/sitio_decretos/1normas.htm. Consultado Diciembre de 2004.

15. Departamento Administrativo Nacional de Estadística DANE. Bogotá. Proyecciones de población por sexo y edad, 1985 - 2006. Bogotá: Ediciones del DANE; 1999.

16. Abramson JH, Abramson ZH. Making sense of data. A self-instruction manual on the interpretation of epidemiological data. 3rd edition. New York: Oxford University Press; 2001. p. 10.

17. Chatfield C. The analysis of time series. An introduction. Boca Ratón: Chapman \& Hall/CRC; 1999. p. 9-26.

18. Singh GK, Yu SM. US childhood mortality, 1950 through 1993: trends and socioeconomic differentials. Am J Public Health 1996; 86: 505-12.

19. Sánchez R, Orejarena S, Guzmán Y. Características de los suicidas en Bogotá: 1985 - 2000. Revista de Salud Pública 2004; 6(3);217-234. 